\title{
Unidade de Terapia Intensiva Pediátrica: perfil das internações e mortalidade
}

\section{Pediatric Intensive Care Unit: profile of hospitalizations and mortality}

\author{
Marilian Bastiani Benetti, Angela Regina Maciel Weinmann, \\ Luciane Flores Jacobi, Anaelena Bragança de Moraes
}

Como citar este artigo: BENETTI, MARILIAN B.; WEINMANN ANCELA R. M.; JACOBI, LUCIANE F; MORAES, ANAELENA B.

Unidade de Terapia Intensiva Pediátrica: perfil das internacões e mortalidade. Revista Saúde (Sta. Maria). 2020; 46 (1).

\section{Autor correspondente:}

Nome: Marilian Bastiani Benetti

E-mail: marilianbbenetti@gmail.com

Telefone: (55) 9987-7037

Formação Profissional: Mestre

em Ciências da Saúde pela

Universidade Federal de Santa

Maria (UFSM) que fica na cidade

de Santa Maria, RS, Brasil.

Filiação Institucional: Anesped Clinica Integrada LTDA

Endereço para correspondência:

Rua: Fernando Ferrari

Bairro: Nossa Senhora de Lourdes

Cidade: Santa Maria

Estado: Rio Grande do Sul

CEP: 97050-80 I

Data de Submissão:

$31 / 10 / 2019$

Data de aceite:

01/04/2020

Conflito de Interesse: Não há conflito de interesse

\section{(cc) $\mathrm{BY}-\mathrm{NC}-\mathrm{ND}$}

\section{RESUMO}

Objetivo: Caracterizar e avaliar associações das variáveis relacionadas às internações e mortalidade em uma Unidade de Terapia Intensiva Pediátrica (UTIP) de um Hospital Universitário no período de 2006 a 2013. Método: Trata-se de um estudo transversal, descritivo e retrospectivo, sendo avaliados dados de prontuários de 1805 internações ocorridas na UTIP, num período de 8 anos. Resultados: Das 1805 internações na UTIP nesse período, 56,1\% eram do sexo masculino, sendo 42,4\% procedentes da cidade de Santa Maria. A maior frequência foi de pacientes com até um ano de idade $(41,6 \%)$, sendo a idade mais frequente de dois meses. 0 tempo médio de permanência na UTIP foi de 7,5 dias. As causas de internação mais frequentes foram: pneumonia, pós-operatório abdominal, trauma e sepse. A mortalidade foi de $14,3 \%$ (sendo $42,6 \%$ por sepse, 15,3\% por pneumonia, $8 \%$ por trauma e 2,8\% por pós-operatório abdominal) e do total de pacientes que internaram, 85,7\% tiveram alta da UTIP. A proporção de mortes por sepse foi significativamente maior do que a por trauma e pós-operatório abdominal, mas não diferiu da mortalidade por pneumonia. Conclusão: Houve predominância das internações em crianças de dois meses de idade sendo sepse a principal causa de mortalidade.

PALAVRAS-CHAVE: Perfil de saúde; Unidades de Terapia Intensiva; Pediatria; Hospitais Universitários.

\section{ABSTRACT}

Objective: To characterize and evalute associations of variables related to hospitalizations and the mortality in a Pediatric Intensive Care Unit (PICU) of a University Hospital from 2006 to 2013. Method: It was developed a descriptive, retrospective and cross sectional study with investigations of 1805 patient records from hospitalizations occurred in 8 years in the PICU. Results: Hospital discharge was observed in $87,5 \%$ of the patients hospitalized which frequently presented age until 1 year old $(41,6 \%)-2$ months old highlighted. The average length of patients' stay was 7 and half days and the main causes of hospitalization were pneumonia, abdominal postoperative, trauma and sepsis. Moreover, the rate of mortality was $14,3 \%$ (42,6\% of it due to sepsis) and the proportion of death by sepsis was significantly higher than trauma and abdominal postoperative - with no further differences from the mortality caused by pneumonia. Conclusion: The hospitalizations of 2 month-old children were predominant and the mortality was most resulted from sepsis.

KEYWORDS: Health profile; Intensive Care Units; Pediatrics; Hospitals University. 


\section{INTRODUÇÃO}

O final da década de 50 marca o início das Unidades de Terapia Intensiva (UTI) ${ }^{1}$, com intuito de salvar a vida de pacientes graves, com risco iminente de morte ${ }^{2}$. Já, o tratamento intensivo para crianças muito enfermas somente a partir da década de 70 mostrou importante evolução, com a criação, nos Estados Unidos e Canadá, das primeiras unidades nos Hospitais Infantis da Filadélfia e de Toronto³.

No Brasil, a primeira UTI foi criada em 1967 e, desde então, tem se observado um crescimento constante nesse tipo de tratamento, especialmente nos hospitais classificados como universitários, devido à complexidade dos pacientes por eles assistidos ${ }^{4}$. O Ministério da Saúde, em sua Portaria no. 7, de 24 de fevereiro de 2010, regulamenta e define as UTIs como "área crítica destinada à internação de pacientes graves, que requerem atenção profissional especializada de forma contínua, materiais específicos e tecnologias necessárias ao diagnóstico, monitorização e terapia".

A Unidade de Terapia Intensiva Pediátrica (UTIP) do Hospital Universitário de Santa Maria (HUSM) foi fundada em 1995, ou seja, 28 anos após o surgimento da primeira UTI no Brasil. Atualmente, é constituída por cinco leitos (sendo dois de isolamento). Atende pacientes clínicos e cirúrgicos, oriundos não só da cidade de Santa Maria, mas também da região a qual o hospital é referência (região da $4^{\text {a }}$ Coordenadoria Regional de Saúde (CRS) onde há mais de um milhão de habitantes) e também recebe pacientes de todo estado do Rio Grande do Sul, por meio da Central Reguladora de Leitos do Estado. É a única UTIP da região centro-sul do estado do RS que atende pacientes exclusivamente do Sistema Único de Saúde (SUS).

Pela importância desta UTIP na região, há interesse em conhecer os dados epidemiológicos de morbimortalidade desta unidade de saúde, permitindo, assim, uma tomada de decisões, visando o aperfeiçoamento da qualidade da atenção prestada. A partir disso, a aquisição de novas tecnologias, o treinamento dos profissionais, a reavaliação dos processos de atenção e a adaptação estrutural podem ser planejados com o objetivo de adequação da unidade às características demográficas e de morbidade da população que a demanda ${ }^{6}$.

Portanto, o presente estudo teve por objetivo caracterizar e avaliar associações das variáveis relacionadas às internações e mortalidade em uma UTIP de um Hospital Universitário no período de 2006 a 2013.

\section{MÉTODO}

Trata-se de um estudo transversal, descritivo e retrospectivo. A população alvo foi composta pelos pacientes que internaram na UTIP de 2006 a 2013. Tanto pacientes pertencentes à $4^{\mathrm{a}}$ CRS do estado do Rio Grande do Sul - Região de Santa Maria, quanto os advindos de qualquer outra região via Central Reguladora de Leitos do Estado. 
Cada paciente que interna em uma UTIP tem seu registro realizado, constando as seguintes informações: número do SAME (Serviço de Arquivo Médico e Estatística), sexo, data de nascimento, data da internação, causa da internação (patologia), desfecho (alta, transferência ou óbito), data do desfecho, procedência, necessidade de ventilação mecânica, presença de convulsão, dentre outras. É preenchido, atualmente, de forma manual pelos profissionais atuantes na Unidade ficando sob os cuidados da secretaria da Unidade. A qualidade do preenchimento do Livro de Registros de Internações tem melhorado ao longo do tempo, pelo cuidado nas anotações das informações, justificando assim, a seleção dos dados dos anos mais recentes.

Foram incluídos no estudo todos os dados dos pacientes com registro de internação no período de 2006 a 2013. Para pacientes com mais de uma admissão na Unidade, foi considerada cada internação como independente.

Um protocolo de coleta de dados foi construído, pelas pesquisadoras, constando as informações do registro na internação na UTIP.

Por se tratar de dados secundários, não foi necessário utilizar o Termo de Consentimento Livre e Esclarecido, somente o Termo de Confidencialidade. Esta pesquisa foi aprovada pelo Comitê de Ética em Pesquisa da Universidade Federal de Santa Maria - UFSM, sob o número: 37867414.9.0000.5346.

Durante os oito anos analisados, houve uma demanda de 1805 internações na UTIP, resultando na população deste estudo. $\mathrm{O}$ banco de dados para a análise foi construído utilizando-se uma planilha no Microsoft Excel囚. As variáveis consideradas foram: sexo, idade (obtida através do cálculo da data de internação menos a data do nascimento e registrada em meses), procedência (procedente de Santa Maria, pertencente à Região da $4^{\mathrm{a}} \mathrm{CRS}$ ou fora dessa área de abrangência), causa da internação, desfecho (óbito ou alta (transferência para outra unidade ou alta para o domicílio)) e tempo de internação (data do desfecho menos a data da internação).

Foi realizada a análise estatística descritiva e inferencial não paramétrica, utilizando-se os testes: de normalidade de Shapiro-Wilk, U de Mann-Whitney, Qui-Quadrado de Aderência, de Independência e Análise de Resíduos. Todas as análises foram realizadas utilizando-se o aplicativo computacional Statistica 9.1, considerando um nível de significância de $5 \%$.

\section{RESULTADO}

No período de 2006 a 2013, das 1805 admissões na UTIP do HUSM 56,1\% (1012) eram de crianças do sexo masculino e 43,9\% (793) do sexo feminino, sendo uma prevalência significativamente maior de internações de meninos (p-valor < 0,01). Quanto à procedência, 42,4\% (758) eram de Santa Maria, 27,4\% (491) de outra cidade da $4^{\mathrm{a}} \mathrm{CRS}$, área 
de abrangência do HUSM, 30,2\% (541) de outra localidade fora da área da $4^{\mathrm{a}}$ CRS sendo 0,8\% (15) internações sem essa informação.

Considerando o número de internações mensais na UTIP, não houve diferença significativa quando comparados todos os meses do período do estudo, ou seja, esses ocorreram de maneira uniforme, sendo o mínimo de 127 internações em fevereiro e o máximo de 178 em agosto. O mesmo foi observado quando avaliado o número de internações anuais, sendo 2008 o ano com menor número de internações (198) e 2012 com o maior número (263).

O tempo médio das internações foi de 7,5 dias (desvio padrão = 80,3 dias), sendo o mínimo de 1 dia e máximo de 9,3 anos. Ainda, $50 \%$ das internações foram de 3 dias ou mais (mediana $=3$ dias) e $10 \%$ das internações foram de 13 dias ou mais. Um único paciente portador de distrofia muscular permaneceu mais de nove anos (9,3 anos) internado na unidade, devido à necessidade de cuidados especiais, considerando que o hospital não conta com unidade de cuidados intermediários pediátricos.

Quanto à idade dos pacientes internados, 41,6\% tinha até um ano, sendo a idade mais frequente a de dois meses. A média das idades foi de 4,3 anos (desvio padrão = 4,7anos) e a mediana de dois anos (mínima de um mês e máxima de 26,5 anos). A média de idade dos meninos foi de 4,2 anos (desvio padrão = 4,7 anos) e mediana de 1,8 anos (mínimo de 1 mês e máximo de 22,2 anos) e para as meninas a média de idade foi 4,3 anos (desvio padrão = 4,7 anos) e mediana de 2,2 anos (mínimo 1 mês e máximo de 26,5 anos), não havendo diferença significativa nas idades entre meninos e meninas $(p=$ 0,358). Em relação ao desfecho da internação, 14,3\% (258 pacientes) foram a óbito, 77,3\% (1395) foram transferidos para outra Unidade, 8,4\% (151) receberam alta para o domicílio não constando o desfecho para uma das internações.

O percentual de óbitos oscilou de 11,0\% em 2012 a 17,6\% em 2009, não havendo diferença significativa (p-valor $=0,303$ ) nas proporções de óbitos entre os anos avaliados (FIGURA 1).

Figura 1. Distribuição da proporção de óbitos na UTIP do HUSM, de 2006 a 2013.

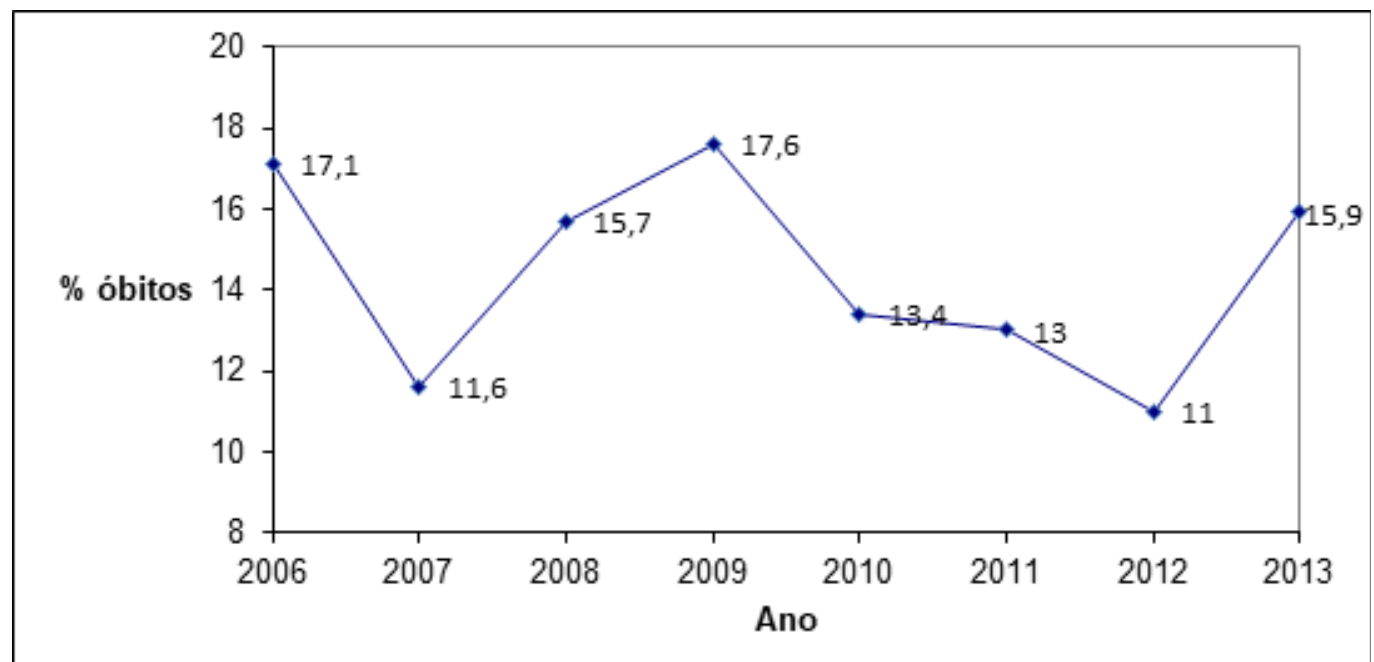


A proporção de óbitos de meninas $(16,7 \%)$ foi significativamente maior ( $p$-valor $=0,011$ ) do que a de meninos $(12,4 \%)$, no período de 8 anos. Não houve diferença significativa ( $p$-valor $=0,380)$ na mortalidade por faixa etária (FIGURA2).

Figura 2. Distribuição da mortalidade, por faixa etária, dos pacientes que internaram na UTIP do HUSM de 2006 a 2013

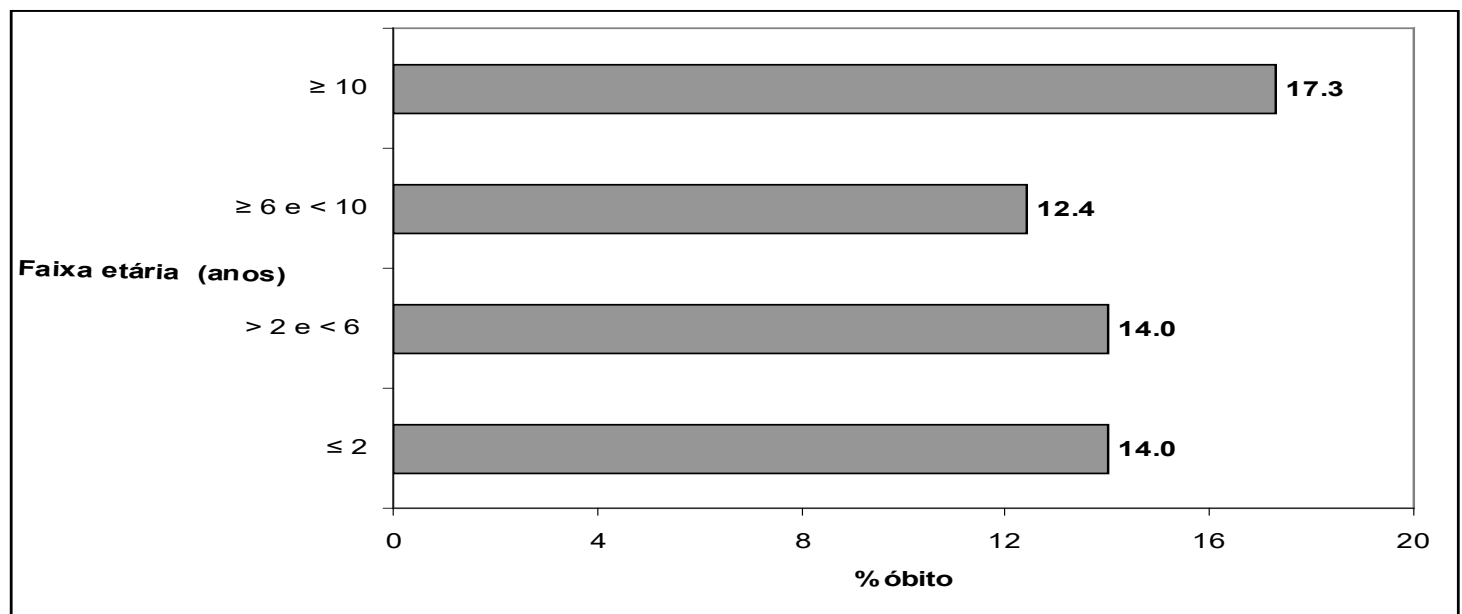

Os tempos de internação dos pacientes que foram a óbito (média=5,5 dias) foram significativamente menores em relação aos que tiveram alta (média $=19,8$ dias $)(p$-valor $=0,023)$.

Na Tabela 1 são apresentados os números de internações e os percentuais de óbitos distribuídos segundo a causa da internação e o sexo dos pacientes.

Pode-se observar na Tabela 1 que as causas de internação mais frequentes foram: pneumonia, pós-operatório abdominal, trauma e sepse. A mortalidade relativa dessas causas de internação (independente do sexo) foi de $42,6 \%$ para sepse, 15,3\% para pneumonia, 8\% para trauma e 2,8\% para pós-operatório abdominal. Quando comparadas essas causas mais frequentes de internação, houve diferença significativa ( $p$-valor $<0,01$ ) na mortalidade, sendo que a mortalidade por sepse foi significativamente maior do que a mortalidade no pós-operatório abdominal e trauma, mas não diferiu da mortalidade por pneumonia.

Agora, considerando somente a mortalidade, independente da causa de internação, os maiores percentuais de óbito ocorreram, sequencialmente, para sepse $(42,2 \%)$, leucemia $(40,4 \%)$, insuficiência cardíaca $(33,3 \%)$, outros problemas neurológicos $(31,6 \%)$ e outros tumores $(30,7 \%)$. 
Tabela 1. Número de internações e percentual de óbitos em função da causa da internação e sexo, na UTIP do HUSM de 2006 a 2013.

\begin{tabular}{|c|c|c|c|c|c|c|}
\hline \multirow[b]{3}{*}{ Causa da internação } & \multicolumn{4}{|c|}{ Sexo } & \multirow{2}{*}{\multicolumn{2}{|c|}{ Total }} \\
\hline & \multicolumn{2}{|c|}{ Masculino } & \multicolumn{2}{|c|}{ Feminino } & & \\
\hline & $\mathbf{n}$ & \%óbito & $\mathbf{n}$ & \%óbito & $\mathbf{n}$ & \%óbito \\
\hline \multicolumn{7}{|l|}{ Problemas cardiocirculatórios } \\
\hline Sepse/choque séptico & 76 & 40,8 & 45 & 44,4 & 121 & 42,2 \\
\hline Insuficiência cardíaca & 4 & 50,0 & 5 & 20,0 & 9 & 33,3 \\
\hline Cardiopatia congênita & 28 & 25,0 & 31 & 19,4 & 59 & 22,0 \\
\hline Outros & 4 & 0,0 & 4 & 25,0 & 8 & 12,5 \\
\hline \multicolumn{7}{|l|}{ Problemas hematológicos } \\
\hline Leucemia & 27 & 33,3 & 30 & 46,7 & 57 & 40,4 \\
\hline Outros tumores & 35 & 25,7 & 40 & 35,0 & 75 & 30,7 \\
\hline \multicolumn{7}{|l|}{ Pós-operatório } \\
\hline Abdominal & 136 & 1,5 & 74 & 4,1 & 210 & 2,4 \\
\hline Neurológico & 10 & 0,0 & 9 & 11,1 & 19 & 5,3 \\
\hline Outros & 21 & 0,0 & 24 & 0,0 & 45 & 0,0 \\
\hline \multicolumn{7}{|l|}{ Causas externas } \\
\hline Trauma & 109 & 6,4 & 52 & 9,6 & 161 & 7,4 \\
\hline Intoxicação exógena & 10 & 0,0 & 14 & 7,4 & 24 & 4,2 \\
\hline Outros & 28 & 3,6 & 14 & 14,3 & 42 & 7,1 \\
\hline \multicolumn{7}{|l|}{ Problemas gastrointestinais } \\
\hline Gastroenterites/diarréias & 19 & 0,0 & 11 & 27,3 & 30 & 10,0 \\
\hline Outros & 20 & 0,0 & 16 & 37,5 & 36 & 16,7 \\
\hline \multicolumn{7}{|l|}{ Problemas metabólicos } \\
\hline Diabetes/CAD & 12 & 0,0 & 16 & 6,2 & 28 & 3,6 \\
\hline Distúrbio hidroeletrolítico & 1 & 0,0 & 4 & 0,0 & 5 & 0,0 \\
\hline EIM/Testes & 26 & 3,8 & 27 & 11,1 & 53 & 7,6 \\
\hline \multicolumn{7}{|l|}{ Problemas neurológicos } \\
\hline Meningite/Encefalite & 35 & 11,4 & 35 & 17,1 & 70 & 14,3 \\
\hline $\begin{array}{l}\text { Convulsões/Mal convulsivo } \\
\text { /Epilepsia }\end{array}$ & 45 & 2,2 & 33 & 12,1 & 78 & 6,4 \\
\hline Outros & 35 & 34,3 & 22 & 27,3 & 57 & 31,6 \\
\hline \multicolumn{7}{|l|}{ Problemas respiratórios } \\
\hline Asma & 6 & 0,0 & 10 & 10,0 & 16 & 6,2 \\
\hline Pneumonia & 183 & 14,8 & 177 & 15,8 & 360 & 15,3 \\
\hline Laringite & 6 & 16,7 & 3 & 0,0 & 9 & 11,1 \\
\hline Bronquiolite & 32 & 0,0 & 17 & 0,0 & 49 & 0,0 \\
\hline Outros & 39 & 15,4 & 38 & 7,9 & 77 & 11,7 \\
\hline Problemas neonatais & 8 & 37,5 & 8 & 12,5 & 16 & 5,9 \\
\hline Alterações renais & 21 & 4,8 & 13 & 7,7 & 34 & 5,9 \\
\hline Problemas dermatológicos & 23 & 8,7 & 14 & 7,1 & 37 & 8,1 \\
\hline Total & 999 & & 787 & & $1786^{*}$ & 14,5 \\
\hline
\end{tabular}

*Das 1805 internações, 19 não constavam a causa de internação. 
Na Tabela 2 são apresentados os números de internações e o percentual de óbitos pela causa da internação e faixa etária.

Tabela 2. Número de internações e percentual de óbitos em função da causa da internação e faixa etária, na UTIP do HUSM de 2006 a 2013.

\begin{tabular}{|c|c|c|c|c|c|c|c|c|}
\hline \multirow[b]{3}{*}{ Causa da internação } & \multicolumn{8}{|c|}{ Faixa etária } \\
\hline & \multicolumn{2}{|c|}{$\begin{array}{l}\text { Lactente } \\
\text { até } 2 \text { anos }\end{array}$} & \multicolumn{2}{|c|}{$\begin{array}{l}\text { Pré-escolar } \\
>2 \text { e }<6 \text { anos }\end{array}$} & \multicolumn{2}{|c|}{$\begin{array}{l}\text { Escolar } \\
\geq 6 \text { e }<10 \text { anos }\end{array}$} & \multicolumn{2}{|c|}{$\begin{array}{l}\text { Adolescente } \\
\geq 10 \text { anos }\end{array}$} \\
\hline & $\mathbf{n}$ & \%óbito & $\mathbf{n}$ & \%óbito & $\mathbf{n}$ & \%óbito & $\mathbf{n}$ & \%óbito \\
\hline \multicolumn{9}{|l|}{ Problemas cardiocirculatórios } \\
\hline Sepse/choque séptico & 78 & 39,7 & 18 & 55,6 & 12 & 16,7 & 13 & 61,5 \\
\hline Insuficiência cardíaca & 4 & 25,0 & 1 & 0,0 & 0 & 0,0 & 4 & 50,0 \\
\hline Cardiopatia congênita & 47 & 17,0 & 6 & 33,3 & 3 & 66,7 & 3 & 33,3 \\
\hline Outros & 3 & 0,0 & 1 & 0,0 & 1 & 0,0 & 3 & 33,3 \\
\hline \multicolumn{9}{|l|}{ Problemas hematológicos } \\
\hline Leucemia & 8 & 62,5 & 13 & 38,5 & 18 & 27,8 & 18 & 44,4 \\
\hline Outros tumores & 16 & 25,0 & 23 & 39,1 & 16 & 31,2 & 20 & 25,0 \\
\hline \multicolumn{9}{|l|}{ Pós-operatório } \\
\hline Abdominal & 119 & 0,8 & 49 & 2,0 & 13 & 7,7 & 28 & 7,1 \\
\hline Neurológico & 6 & 16,7 & 8 & 0,0 & 5 & 0,0 & 0 & 0,0 \\
\hline Outros & 13 & 0,0 & 7 & 0,0 & 8 & 0,0 & 17 & 0,0 \\
\hline \multicolumn{9}{|l|}{ Causas externas } \\
\hline Trauma & 22 & 4,6 & 30 & 10,0 & 47 & 6,4 & 62 & 8,1 \\
\hline Intoxicação exógena & 4 & 25,0 & 11 & 0,0 & 4 & 0,0 & 5 & 0,0 \\
\hline Outros & 9 & 11,1 & 10 & 10,0 & 14 & 7,1 & 9 & 0,0 \\
\hline \multicolumn{9}{|l|}{ Problemas gastrointestinais } \\
\hline Gastroenterites/diarréias & 23 & 8,7 & 2 & 50,0 & 2 & 0,0 & 3 & 0,0 \\
\hline Outros & 10 & 0,0 & 6 & 0,0 & 12 & 25,0 & 8 & 37,5 \\
\hline \multicolumn{9}{|l|}{ Problemas metabólicos } \\
\hline Diabetes/CAD & 2 & 50,0 & 5 & 0,0 & 12 & 0,0 & 9 & 0,0 \\
\hline Distúrbio hidroeletrolítico & 0 & 0,0 & 0 & 0,0 & 2 & 0,0 & 3 & 0,0 \\
\hline EIM/Testes & 34 & 8,8 & 9 & 0,0 & 5 & 0,0 & 5 & 20,0 \\
\hline \multicolumn{9}{|l|}{ Problemas neurológicos } \\
\hline Meningite/Encefalite & 40 & 12,5 & 10 & 10,0 & 11 & 18,2 & 9 & 22,2 \\
\hline $\begin{array}{l}\text { Convulsões/Mal } \\
\text { convulsivo/Epilepsia }\end{array}$ & 33 & 6,1 & 19 & 0,0 & 13 & 7,7 & 13 & 15,4 \\
\hline Outros & 27 & 44,4 & 8 & 25,0 & 9 & 22,2 & 13 & 15,4 \\
\hline \multicolumn{9}{|l|}{ Problemas respiratórios } \\
\hline Asma & 7 & 0,0 & 4 & 0,0 & 3 & 33,3 & 2 & 0,0 \\
\hline Pneumonia & 252 & 13,9 & 61 & 13,1 & 22 & 18,2 & 26 & 30,8 \\
\hline Laringite & 5 & 20,0 & 3 & 0,0 & 1 & 0,0 & 0 & 0,0 \\
\hline Bronquiolite & 49 & 0,0 & 0 & 0,0 & 0 & 0,0 & 0 & 0,0 \\
\hline Outros & 60 & 11,7 & 6 & 33,3 & 4 & 0,0 & 7 & 0,0 \\
\hline Problemas neonatais & 15 & 6,7 & 1 & 0,0 & 0 & 0,0 & 0 & 0,0 \\
\hline Alterações renais & 4 & 0,0 & 2 & 0,0 & 12 & 0,0 & 16 & 12,5 \\
\hline Problemas dermatológicos & 14 & 14,3 & 6 & 0,0 & 13 & 7,7 & 4 & $\overline{0,0}$ \\
\hline Total & 904 & & 319 & & 262 & & 300 & \\
\hline
\end{tabular}

*Das 1805 internações, 19 não constavam a causa de internação e uma internação sem informação de idade. 
Considerando as informações da Tabela 2 e a mesma seleção das causas de internação mais frequentes (pneumonia, pós-operatório abdominal, trauma e sepse), os percentuais de óbitos por faixa etária não diferiram significativamente ( $p$-valor > 0,05) para essas causas. Pode-se observar que 50,6\% (904) das 1785 internações foram de crianças (lactentes), ou seja, menores de dois anos de idade (Tabela 2).

Na Figura 3 são apresentados os percentuais de óbitos por grupo de causas da internação na UTIP.

Figura 3. Distribuição do número de internações por grupo de causas e desfecho na UTIP do HUSM, 2006 a 2013.

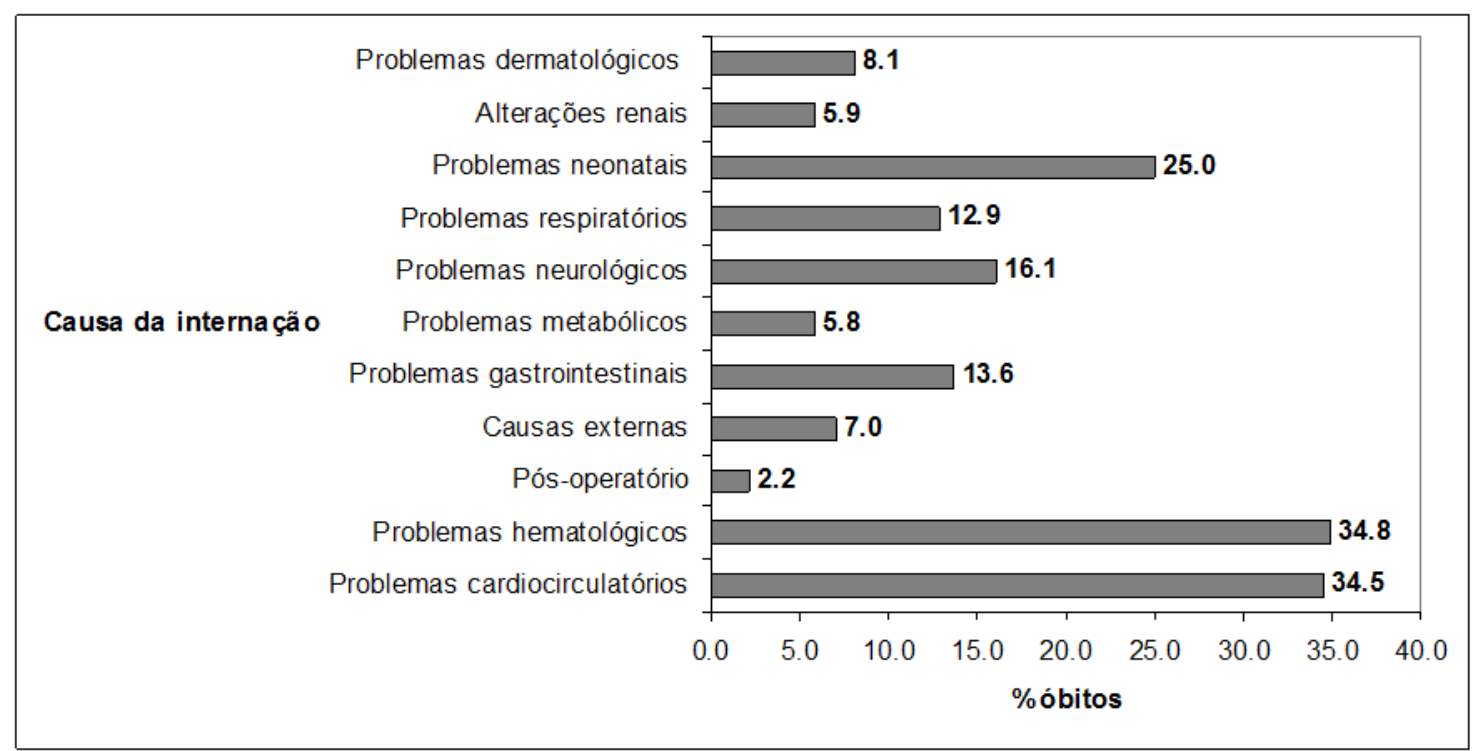

Na comparação dos óbitos de todos os grupos de causas, houve significativamente mais óbitos do que o esperado ao acaso para as causas de internação por problemas cardiocirculatórios e hematológicos em relação às outras causas e menos óbitos do que esperados ao acaso para pós-operatório, causas externas e problemas metabólicos (Figura 3).

\section{DISCUSSÃO}

O conhecimento de dados epidemiológicos da população atendida em uma unidade de terapia intensiva é tão importante quanto o investimento em novos recursos de tratamento e de tecnologias de ponta ${ }^{7}$.

Estudos realizados com informações obtidas de dados secundários têm o benefício de serem de domínio público. Por outro lado, esses dados podem não ser inteiramente fidedignos e não mostrarem integralmente a realidade da população, pois além do sub-registro, as informações são obtidas por diferentes processos de trabalho e profissionais.

Mesmo assim, estudos epidemiológicos deste tipo são fundamentais, pois permitem conhecer estatísticas de UTIP brasileiras com o objetivo de programar ações para melhorias. A UTIP do HUSM, objeto deste estudo, é centro de referência para crianças gravemente enfermas, abrangendo uma região de mais de um milhão de habitantes pertencentes 
aos 32 municípios da $4^{\text {a }}$ CRS. Possui aparato tecnológico de alta complexidade, profissionais qualificados e atualizados para atuar com crianças criticamente doentes.

No período do presente estudo, compreendido entre os anos de 2006 a 2013, o que representa quase metade do tempo de existência desta unidade, foram avaliados os dados referentes a todas as internações ocorridas, totalizando 1805. Este número é pequeno se comparado ao número de internações mensais que ocorrem no Serviço de Pediatria do HUSM. Tendo em vista o grande fluxo de pacientes que chegam ao Serviço, deve-se considerar que a população estudada é representada pelas doenças mais severas, que necessitam de cuidados em Unidades de Terapia Intensiva, por equipes especializadas.

Considerando as internações que ocorreram, a maioria $56,1 \%$ foi do sexo masculino, estando de acordo com outros estudos $4,6,8,9,10,11$ realizados em UTIP de alguns estados brasileiros, que também encontraram maior prevalência do sexo masculino.

Quanto à idade, os resultados do presente estudo corroboram com os estudos de Costa et al. ${ }^{4}$, Alves et al. ${ }^{10} \mathrm{e}$ Veras et al. ${ }^{12}$. A média e a mediana das idades encontradas se assemelham ao estudo de Lanetzkiet al. ${ }^{6}$ bem como a prevalência de menores de um ano.

Quanto à procedência dos pacientes que internaram, 42,4\% eram de Santa Maria e 57,6\% de outras localidades pertencentes à área da $4^{\mathrm{a}} \mathrm{CRS}$ e mesmo de localidades fora desta abrangência (internação solicitada por meio da Central de Leitos do Estado). Esse resultado diferiu do encontrado no trabalho de Veras et al. ${ }^{12}$ onde $89,2 \%$ era procedente da cidade do estudo, porém teve semelhança com o estudo de Alves et al.10, em que 74\% não eram naturais da cidade.

O número das internações não diferiu significativamente entre os meses nem entre os anos, sendo o número máximo em agosto. Poucos estudos ${ }^{6,10,12}$ avaliaram a sazonalidade das internações, encontrando, também, o mês de agosto como o de maior número de internações.

O tempo médio que os pacientes permaneceram internados foi de aproximadamente 7,5 dias, corroborando com outros estudos ${ }^{4,8,9,11}$. Já Alves et al. ${ }^{10}$ encontraram tempo médio de permanência um pouco menor, 5,4 dias, concluindo que isso se deveu à alta rotatividade dos pacientes na unidade, pois o setor recebe muitos casos de pós-operatório imediato. Em contraponto, o estudo de Mendonça et al. ${ }^{11}$ não se restringiu à análise das internações de somente uma UTIP, abrangendo o conjunto da rede do SUS no estado de Pernambuco, de modo a revelar permanência média de 14,4 dias, com valor aumentado $(18,7)$ para pacientes com idade menor que 1 ano.

Em relação ao desfecho da internação, 14,3\% foram a óbito. Esse resultado se mostrou superior ao encontrado em alguns estudos ${ }^{6,8,10}$. A mortalidade oscilou de 11\% em 2012 a 17,7\% em 2009, sendo que não houve diferença significativa na mortalidade geral entre os anos avaliados. Já Costa et al. ${ }^{4}$ encontraram $33,9 \%$ de mortalidade, um número bastante superior ao encontrado no presente estudo. Marcin et al. ${ }^{13}$ referem que a mortalidade é uma importante 
informação para a programação e cuidados na UTIP. Justifica que avaliações precisas em UTIP permitem programas de tratamento apropriados e qualidade nos cuidados.

Considerando as características de cada local e as diferenças regionais, a manutenção contínua de uma avaliação da mortalidade é de fundamental importância para a busca por menores taxas possíveis.

No presente estudo, não foi encontrado diferença significativa entre a mortalidade por faixa de idade, já Einloft et al. ${ }^{8}$ observaram diferença significativa quanto a mortalidade para a faixa etária de menores de um ano. Relatam ainda que a taxa de mortalidade encontrada foi inversamente proporcional à faixa etária, ou seja, quanto mais jovem, maior a mortalidade.

A maior parte das causas de internações neste estudo foi devido a patologias clínicas, sendo elas: pneumonia, pós-operatório abdominal, trauma e sepse, corroborando com o resultado do estudo realizado por Alves et al. ${ }^{10}$. Em estudo referente às internações por causas respiratórias em duas UTIP de Salvador (BA), Andrade et al..$^{14}$ também determinaram que a principal causa de admissão foi a pneumonia.

A maior mortalidade foi por sepse/choque séptico seguida por causas respiratórias (pneumonia). Outros estudos também encontraram sepse como sendo a principal causa de mortalidade ${ }^{4,8,15}$, sendo que outros encontraram como causas principais de óbito as afecções do aparelho respiratório ${ }^{9,10}$ e as doenças infecto-parasitárias ${ }^{11}$.

Lanetzki et al. ${ }^{6}$ observaram predominância de causas respiratórias nas internações, sendo a pneumonia a mais frequente. Como causa de óbito, o resultado diferiu do encontrado neste estudo, pois as neoplasias foram as causas principais, podendo ser devido a viés, uma vez que houve somente oito óbitos no estudo.

\section{CONSIDERAÇÕES FINAIS}

As características identificadas neste estudo são semelhantes às de outras UTIP, tais como maior prevalência de internações do sexo masculino, de internações por causas respiratórias, de mortalidade por sepse/choque séptico, assim como o tempo médio de internação ser pouco maior do que uma semana.

O estudo realizado apresentou limitações quanto a obtenção dos dados, pois os mesmos foram retirados de prontuários hospitalares. Dessa maneira, não se pode descartar a possibilidade de erros referentes às causas de internação e as causas básicas dos óbitos.

Considerando a limitação da amostra os resultados desse estudo devem ser interpretados com cuidado quando utilizados como referência para outras populações.

Conhecendo as particularidades da população internada, da mesma forma que as condições clínicas e as circunstâncias em que os óbitos ocorreram, tem-se por perspectivas prever recursos, organizar processos e treinar profissionais para melhorar os cuidados dispensados àqueles que são encaminhados à unidade de cuidados intensivos, 
seja proporcionando maior conforto e melhorando a qualidade de vida dos pacientes, seja atenuando o sofrimento daqueles para os quais este é impreterível.

\section{REFERÊNCIAS}

1. Vicent J-L. Where have we been and where are we going? Critical Care 2013; 17(Suppl 1:S2): 1-6.

2. Silva NR, Menezes RA. "Se parar, parou": categorização do morrer em uma unidade de terapia intensiva da cidade do Rio de Janeiro. Physis Revista de Saúde Coletiva, 2015; 25(1): 265-285.

3. Cutulo LR, Furtado Junior JR, Botelho L. Perfil dos pacientes internados na unidade de terapia intensiva pediátrica do hospital infantil Joana de Gusmão no ano de 1993. ACM Arq Catarin Med. 1994; 23(2): 95-100.

4. Costa CMS, Prazeres JS, Rolim JM, Forte SR, De Aquino DMC. Perfil epidemiológico dos pacientes internados em uma Unidade de Terapia Intensiva pediátrica, São Luiz - MA. Rev Hosp Universitário. 2009; 10(3): 61-6.

5. Brasil. Ministério da Saúde. Portaria MS nº 7, de 24 de fevereiro de 2010. [S.I.: s.n.]. [acesso em: 15 jan 2020]. Disponível em: http://bvsms.saude.gov.br/bvs/saudelegis/anvisa/2010/res0007_24_02_2010.html

6. Lanetzki CS, Oliveira CAC, Bass LM, Abramovici S, Troster EJ. O perfil epidemiológico do Centro de Terapia Intensiva Pediátrico do Hospital Israelita Albert Einstein. Rev Einstein. 2012; 10(1): 16-21.

7. Nascimento MSM, Nunes EM, Medeiros RC, Souza WIM, Sousa Filho LF, Alves ESRC. Perfil epidemiológico de pacientes em unidade de terapia intensiva adulto de um hospital regional paraibano. Temas em Saúde. $2018 ; 18(1): 247-265$.

8. Einloft PR, Garcia PC, Piva JP, Bruno F, Kipper DJ, Fiori RM. Perfil epidemiológico de dezesseis anos de uma unidade de terapia intensiva pediátrica. Rev Saúde Pública. 2002; 36(6): 728-33.

9. Batista NOW, Coelho MCR, Trugilho SM, Pinasco GC, Santos EFSS, Ramos-Silva V. Clinical-epidemio- 
logical profile of hospitalised patients in paediatric intensive care unit. Journal of Human Growth and Development. 2015; 25(2): 187-93.

10. Alves MVMFF, Bissiguini PO, Nitsche MJT, Olbrich SRLR, Luppi CHB, Toso LAR. Perfil dos pacientes internados em uma unidade de terapia intensiva pediátrica de um hospital escola do interior de São Paulo. Ciência, Cuidado e Saúde. 2014; 13(2): 294-301.

11. Mendonça JG, Guimarães MJB, de Mendonça VG, Portugal JL, de Mendonça CG. Perfil das internações em Unidades de Terapia Intensiva Pediátrica do Sistema Único de Saúde no estado de Pernambuco, Brasil. Ciênc. saúde coletiva. 2019; 24(3): 907-916.

12. Veras TN, Sandim G, Mundim K, Petrauskas R, Cardoso G, D’Agostin J. Perfil epidemiológico de pacientes pediátricos internados com pneumonia. Porto Alegre: Sci Med. 2010; 20: 277-81.

13. Marcin JP, Pollack MM, Patel KM, Sprague BM, Ruttimann UE. Prognostication and certainty in the pediatric intensive care unit. Pediatrics. 1999; 104(4): 868-873.

14. Andrade VND, Amoretti CF, de Araújo Torreão L, Teixeira e Sousa I. Perfil das internações por causas respiratórias em duas unidades de terapia intensiva pediátricas em Salvador, Bahia. Revista Baiana de Saúde Pública. 2016; 40(1): 250-262.

15. Marcin JP, Pollack MM, Patel KM et al. Combining physician's subjective and physiology-based objective mortality risk predictions. Crit Care Med. 2000; 28: 2984-90. 